\title{
A control scheme research based on sliding mode and proportional-integral control for three-phase rectifier
}

\author{
Wu Chen ${ }^{\text {a) }}$ Li Yinghui, and Liu Cong \\ Aeronautics and Astronautics Engineering School, Air Force Engineering \\ University, Xian, Shaanxi, China \\ a)fxltsbl1028@126.com
}

\begin{abstract}
In order to solve the problem of classic control methods in slow transient response and low anti-interference capacity, this paper proposed a dual closed-loop control scheme based on sliding mode (SM) and proportional-integral (PI) control for a three-phase rectifier. In this work, the error between $i_{d}, i_{q}$ and their desired value were treated as the states of the sliding mode surface, considering the control objectives of power factor and output voltage, which improved the stability and robust performance; besides, a double power reaching law was applied in the controller, which improved the dynamic performance, as well as restraining the chatter. Finally, the proposed strategy was tested in simulation and experiment compared with dual closeloop PI-PI scheme.
\end{abstract}

Keywords: three-phase rectifier, sliding mode control, power reaching law, reaching speed

Classification: Power devices and circuits

\section{References}

[1] J. Huang, et al.: "Sectional direct power control for converter," Trans. China Electrotech. Soc. 31 (2016) 176 (DOI: 10.3969/j.issn.1000-6753.2016.14.020).

[2] Z. Song, et al.: "Direct power control for three-phase two-level voltage-source rectifiers based on extended-state observation," IEEE Trans. Ind. Electron. 63 (2016) 4593 (DOI: 10.1109/TIE.2016.2547859).

[3] L. Dalessandro, et al:: "Center-point voltage balancing of hysteresis current controlled three-level PWM rectifiers," IEEE Trans. Power Electron. 23 (2008) 2477 (DOI: 10.1109/TPEL.2008.2002060).

[4] B. S. Chen and G. Joos: "Direct power control of active filters with averaged switching frequency regulation," IEEE Trans. Power Electron. 23 (2008) 2729 (DOI: 10.1109/TPEL.2008.2004958).

[5] T. Soeiro and J. Kolar: "Analysis of high-efficiency three-phase two- and threelevel unidirectional hybrid rectifiers," IEEE Trans. Ind. Electron. 60 (2013) 3589 (DOI: 10.1109/TIE.2012.2205358).

[6] L. Lai, et al.: "A new PWM approach for digital boost power factor correction controller,” IEICE Electron. Express 12 (2015) 20150279 (DOI: 10.1587/elex. 12.20150279).

[7] H. Li and Y. Li: "Grid-voltage sensorless three-phase PWM rectifier control," 
Power Electron. 49 (2015) 5 (DOI: 10.3969/j.issn.1000-100X.2015.03.002).

[8] X. Zhang, et al.: "dSPACE real-time simulation and controller parameters tuning for three-phase PWM rectifier," Trans. China Electrotech. Soc. 28 (2013) 219 (DOI: 10.3969/j.issn.1000-6753.2013.02.030).

[9] J. H. Kim, et al.: "Direct power control of three-phase boost rectifiers by using a sliding-mode scheme,” J. Power Electron. 13 (2013) 1000 (DOI: 10.6113/ JPE.2013.13.6.1000).

[10] H. Ma, et al:: "A novel exponential reaching law of discrete-time sliding-mode control,” IEEE Trans. Ind. Electron. 64 (2017) 3840 (DOI: 10.1109/TIE.2017. 2652390).

\section{Introduction}

With the intense research of reactive power compensation, harmonic suppression and load shock suppression, three-phase rectifier has a wide application in highvoltage transmission, radio communication, solar and wind power, and its research on control scheme has been a hot spot in power electronics $[1,2]$.

Hysteresis control is an extensively used method for three-phase rectifiers, which can adjust instantaneous current [3] and power [4] through the look-up table. This kind of control scheme has a simple structure and is easy to realize by hardware, but it has the drawback of a variable switching frequency, which will greatly impact the adjustment performance. The control method based on PulseWidth Modulation (PWM) can effectively solve the problem of a variable switching frequency $[5,6]$. In the previous research of three-phase rectifier, proportionalintegral (PI) control most commonly associated with PWM [7]. However, the traditional PI controller is sensitive to the changes of circuit parameters and load; consequently, the system performance will greatly decrease under external disturbance or instant load/unload. Compared with PI control, sliding mode (SM) control has a better robust and dynamic performance, it can decrease the system overshoot when parameters and load change, and reach the stable condition sooner. Literature $[8,9]$ proposed a sliding mode controller in the inner loop to control the instantaneous current and power, which improved the transient response under the load change.

The previous research on SM employed an exponential reaching law, which can improve the dynamic quality by adjusting the parameters [10]; however, as the existence of a constant reaching term, the system will pass across the sliding mode surface at a quick rate, which caused a large chatter. By comparison, in the power reaching law, the reaching speed will fall to zero in the process of reaching the sliding mode surface, which is beneficial to decrease the chatter, but its reaching rate is relatively small during away from the sliding mode surface, which affects the dynamic performance.

In order to enhance the adjusting ability of the reaching speed, this paper proposed a SM-PI control scheme based on a double power reaching law, which maintained a fast speed during away from and closed to the sliding mode surface, and improved the dynamic performance. This control scheme is tested by simulation and experiment finally. 


\section{Controllers design for three-phase rectifier}

When the input voltage stays in ideal balance condition, and the rectifier operates in continuous conduction mode (CCM), its mathematical model in the synchronous rotating frame can be built as below.

$$
\left\{\begin{array}{l}
L \cdot \frac{d i_{d}}{d t}=e_{d}-R i_{d}+\omega L i_{q}-S_{d} V_{d c} \\
L \cdot \frac{d i_{q}}{d t}=e_{q}-R i_{q}-\omega L i_{d}-S_{q} V_{d c} \\
C \cdot \frac{d V_{d c}}{d t}=S_{d} i_{d}+S_{q} i_{q}-\frac{V_{d c}}{R}
\end{array}\right.
$$

Where, $\mathrm{i}_{\mathrm{a}, \mathrm{b}, \mathrm{c}}$ and $\mathrm{e}_{\mathrm{a}, \mathrm{b}, \mathrm{c}}$ are the input current and voltage, $L$ is the filter inductance, $R$ is the equivalent resistance, $C$ is dc-link capacitance, $R$ is the load resistance.

According to the advantage and disadvantage of the previous control scheme for Vienna rectifier, this paper proposed a novel control scheme including a SM inner loop and a PI outer loop (SM-PI control scheme for short).

\subsection{The design of outer loop controller}

The main control objectives of outer loop are tracking the reference voltage and remaining stable, as well as giving the reference value $i_{\mathrm{d}}{ }^{*}$ :

$$
i_{d}^{*}=k_{p}\left(V_{d c}^{*}-V_{d c}\right)+k_{i} \int\left(V_{d c}^{*}-V_{d c}\right) d t
$$

Where, $k_{\mathrm{p}}$ and $k_{\mathrm{i}}$ are the proportional and Integral coefficient in PI controller.

\subsection{The design of inner loop controller}

The main control objectives of inner loop are maintaining the sinusoidal waveform of input current and unity power factor, the desired values are set as $\left[i_{\mathrm{d}}{ }^{*}, i_{\mathrm{q}}{ }^{*}\right]^{\mathrm{T}}$, where $i_{\mathrm{q}}{ }^{*}=0$, the sliding mode surfaces are designed as below.

$$
S_{1,2}=i_{d, \mathrm{q}}^{*}-i_{d, q}
$$

In addition, a double power reaching law were applied to improve the transient performance during the system states are away from the sliding mode surface.

$$
\dot{S}=-k_{1}|S|^{\alpha} \operatorname{sgn}(\mathrm{S})-k_{2}|S|^{\beta} \operatorname{sgn}(\mathrm{S})
$$

Where, $\alpha>1,0<\beta<1$.

Then (5) can be derived as

$$
\left[\begin{array}{c}
S_{d} \\
S_{q}
\end{array}\right]=\frac{1}{V_{d c}}\left[\begin{array}{l}
F_{1}-k_{11} L\left|S_{1}\right|^{\alpha} \operatorname{sgn}\left(\mathrm{S}_{1}\right)-k_{12} L\left|S_{1}\right|^{\beta} \operatorname{sgn}\left(\mathrm{S}_{1}\right) \\
F_{2}-k_{21} L\left|S_{2}\right|^{a} \operatorname{sgn}\left(S_{2}\right)-k_{22} L\left|S_{2}\right|^{\beta} \operatorname{sgn}\left(S_{2}\right)
\end{array}\right]
$$

Where, $F_{1}=e_{d}-R i_{d}+w L i_{q}-L d i_{d}^{*} / d t, F_{2}=e_{q}-R i_{q}-w L i_{d}$.

\subsection{Analysis of sliding mode characteristics}

\subsubsection{Proof of existence and accessibility}

Theorem 1 The system state $S$ can reach the equilibrium $S=0$ under the function of $\dot{S}=-k_{1}|S|^{\alpha} \operatorname{sgn}(S)-k_{2}|S|^{\beta} \operatorname{sgn}(S)$.

\section{Proof}

$$
\dot{S} S=\left[-k_{1}|S|^{\alpha} \operatorname{sgn}(S)-k_{2}|S|^{\beta} \operatorname{sgn}(S)\right] S=-k_{1}|S|^{\alpha+1}-k_{2}|S|^{\beta+1}<0
$$


The above inequation proves that the system satisfies the existence and accessibility condition. Thus, the proof is completed.

\subsubsection{The calculation of reaching time}

Lemma 1 Assume that a radially unbounded function satisfies the following condition: (1) $V(0)=0$, the origin is the global finite time convergence equilibrium; (2) $\exists 0<\mu<1, v>0, r_{\mu}>0, r_{v}>0$, satisfy:

$$
\dot{V} \leqslant \begin{cases}-r_{\mu} V^{1-\mu}, & V \leqslant 1 ; \\ -r_{v} V^{1+v}, & V \geqslant 1 .\end{cases}
$$

Then the origin is the global constant time convergence equilibrium, and the maximum convergence time satisfies:

$$
T_{\max } \leqslant \frac{1}{\mu r_{\mu}}+\frac{1}{v r_{v}}
$$

Theorem 2 For a dynamic model $\dot{S}=-k_{1}|S|^{\alpha} \operatorname{sgn}(S)-k_{2}|S|^{\beta} \operatorname{sgn}(S)$, the state $S$ can converge to zero, and the convergence time exist an upper bound that has no concern with the original state, and

$$
T_{\max }=\frac{1}{k_{1}(\alpha-1)}+\frac{1}{k_{2}(1-\beta)}
$$

Proof $S=0$ is the unique equilibrium of the dynamic model $\dot{S}=$ $-k_{1}|S|^{\alpha} \operatorname{sgn}(S)-k_{2}|S|^{\beta} \operatorname{sgn}(S)$. Then, Lyapunov function is chosen as $V=S^{2}$, its derivative is

$$
\begin{aligned}
\dot{V} & =2 S\left[-k_{1}|S|^{\alpha} \operatorname{sgn}(S)-k_{2}|S|^{\beta} \operatorname{sgn}(S)\right]=-2 k_{1}|S|^{\alpha+1}-2 k_{2}|S|^{\beta+1} \\
& =-2 k_{1} V^{1+(\alpha-1) / 2}-2 k_{2} V^{1-(1-\beta) / 2}
\end{aligned}
$$

And

$$
\dot{V} \leqslant \begin{cases}-2 k_{2} V^{1-(1-\beta) / 2}, & V \leqslant 1 \\ -2 k_{1} V^{1+(\alpha-1) / 2}, & V \geqslant 1\end{cases}
$$

When $\mu=(1-\beta) / 2, v=(\alpha-1) / 2, r_{\mu}=2 k_{2}, r_{v}=2 k_{1}$, the inequation (11) satisfies Lemma 1, and the upper bound of convergence time is $\frac{1}{k_{1}(\alpha-1)}+$ $\frac{1}{k_{2}(1-\beta)}$, which has nothing to do with the original state. Thus, the proof is completed.

\section{Simulation and experimental results}

Here, the effectiveness of the proposed control scheme are verified by a simulation module compared with a dual closed loop PI (PI-PI for short) control scheme. The circuit parameters are shown below: Grid phase voltage $e_{\mathrm{a}, \mathrm{b}, \mathrm{c}}=110 \mathrm{Vrms} / 50 \mathrm{~Hz}$, input inductor $\mathrm{L}=2 \mathrm{mH}$, Equivalent resistance $\mathrm{R}=0.3 \Omega$, dc-link capacitance $\mathrm{C}_{1,2}=2 \mathrm{mF}$, dc-link reference $V_{\mathrm{dc}}{ }^{*}=300 \mathrm{~V}$, Switching frequency $f_{\mathrm{s}}=10 \mathrm{kHz}$. In the dual closed loop SM controller, the parameters of the inner loop are: $k_{11}=$ $k_{12}=k_{21}=k_{22}=4000, \alpha=0.5, \beta=1.5$, the parameters of the outer loop are: $\mathrm{k}_{\mathrm{p}}=0.3, \mathrm{k}_{\mathrm{i}}=10$. In the conventional dual close loop PI controller, the parameters 
of the inner loop are: $\mathrm{k}_{\mathrm{p}}=10, \mathrm{k}_{\mathrm{i}}=5$, the parameters of the inner loop are: $\mathrm{k}_{\mathrm{p}}=0.3, \mathrm{k}_{\mathrm{i}}=10$.

The input current waveforms and the total harmonic distortion (THD) under stable operation are shown in Fig. 1 and Fig. 2.

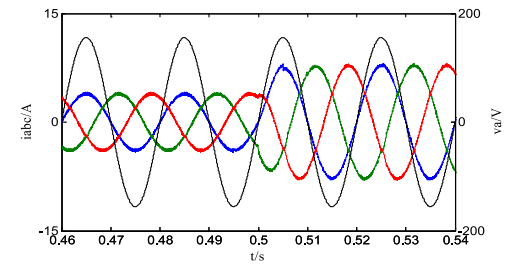

(a) under PI-PI control scheme

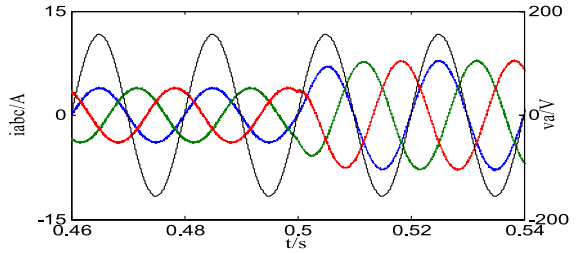

(b) under SM-PI control scheme

Fig. 1. The simulation waveforms of input current and voltage

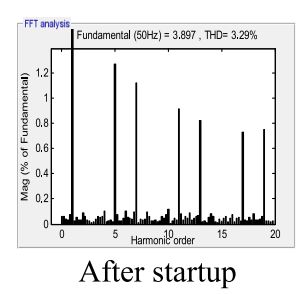

(a) under PI-PI control scheme

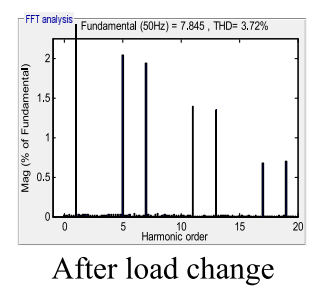

Fig. 2. THD of phase A input phase current

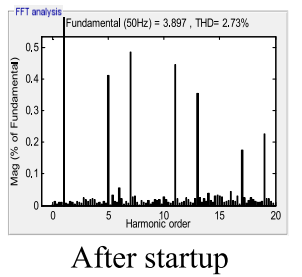

(b) under SM-PI control scheme

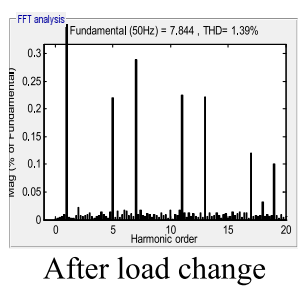

After load change

In this simulation, the load suddenly changes from $100 \Omega$ to $50 \Omega$ at $1.5 \mathrm{~s}$. As is shown in Fig. 1 and Fig. 2, the input current realized sinusoidal waveforms under the two kind of control scheme during the start-up period, however, THD of the phase A input phase current under PI-PI control scheme is a little higher than SM-PI control scheme. What is more, after the load suddenly changes, the former has a large current distortion, and THD is apparently higher than the latter.

As is shown in Fig. 3, under PI-PI control scheme, overshoot after startup is $36.16 \mathrm{~V}$, voltage fluctuation after load change is $-8.18 \mathrm{~V}$, ripple range is $\pm 0.06 \mathrm{~V}$; while under SM-PI control scheme, overshoot after startup is $11.12 \mathrm{~V}$, voltage fluctuation after load change is $-2.54 \mathrm{~V}$, ripple range is $\pm 0.03 \mathrm{~V}$.

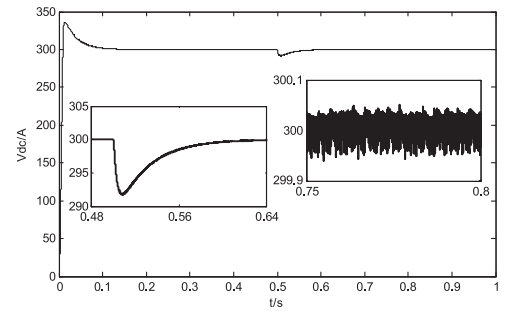

(a) under PI-PI control scheme

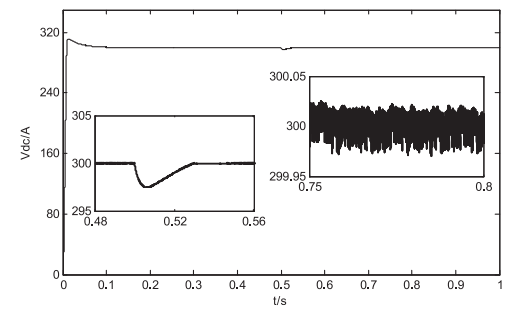

(b) under SM-PI control scheme

Fig. 3. The simulation waveforms of output voltage 
Then, a three-phase Vienna rectifier prototype at the switching frequency of $10 \mathrm{kHz}$ was utilized for the experimental tests, the controller is implemented on TMS320F28377S, and the parameters of circuit and controller are consistent with simulation.

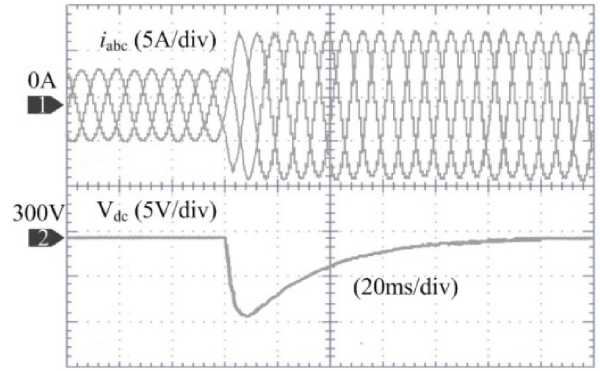

(a) under PI-PI control scheme

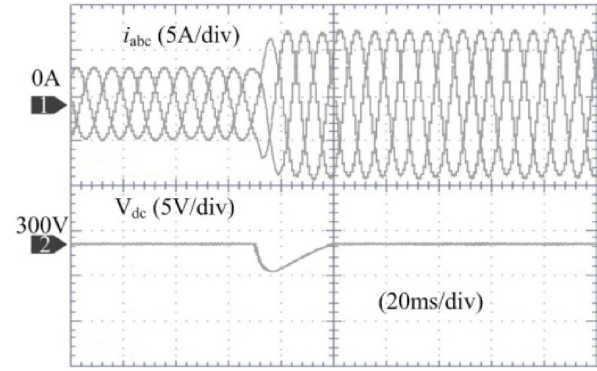

(b) under SM-PI control scheme

Fig. 4. The experiment waveforms of input current and voltage

According to the comparison of input currents in Fig. 4, after the load changed, the rectifier under PI-PI control scheme has a higher current distortion compared with SM-PI control scheme. When the rectifier ran stable again, former's phase A current THD is $5.27 \%$, and latter's is $3.26 \%$, both are inferior to the results of simulations, because the effects of nonideal power device were omitted in the experiment. Besides, after the load changes, the former's fluctuation of output voltage is more than $10 \mathrm{~V}$, and the latter's is less than $4 \mathrm{~V}$, similarly, both were inferior to the results of simulations, because the control system existed time delay in experiment, which has a bad effect on the performance.

Nevertheless, we can draw a same conclusion as the simulation: SM-PI scheme has a better adjusting ability for input current and output voltage, it effectively improved the rectifier's performance of steady state and transient.

\section{Conclusion}

According to the operation feature of three-phase rectifier, a dual close loop SM-PI control scheme was proposed in this paper, the error between $i_{d}, i_{q}$ and their desired value were treated as the states of the sliding mode surface, considering the control objectives of power factor and output voltage; besides, a double power reaching law was applied in the scheme, which improved the dynamic performance, as well as restraining the chatter. From the result in simulation and experiment, the proposed scheme has better characteristics of transient, steady state and robustness compared to PI-PI control scheme. 